\title{
Halogen-bonding-promoted Photo-induced C-X Borylation of Aryl Halide using Phenol Derivatives
}

\author{
Kazuki Matsuo, Eiji Yamaguchi*, Akichika Itoh* \\ Gifu Pharmaceutical University, 1-25-4, Daigaku-Nishi, Gifu, Gifu 501-1196, Japan \\ keyword \\ $C-X$ borylation, aryl halides, halogen bonding complex, photo reaction
}

\begin{abstract}
This study investigates the photo-induced $\mathrm{C}-\mathrm{X}$ borylation reaction of aryl halides by forming a halogen-bonding complex. The method employs 2-naphthol as a halogen-bonding acceptor and proceeds under mild conditions without a photoredox catalyst under $420 \mathrm{~nm}$ blue light irradiation. The method is highly chemoselective, broadly functional group tolerant, and provides concise access to corresponding boronate esters. Mechanistic studies reveal that forming the halogen-bonding complex between aryl halide and naphthol acts as an electron donor-acceptor complex to furnish aryl radicals through photo-induced electron transfer.
\end{abstract}

\section{INTRODUCTION}

Visible light-mediated photoexcitation of small molecules has been used for various useful reactions to specific reactivity derived from electrically excited open-shell chemical species. ${ }^{1}$ On the contrary, direct photochemical activation of small molecules requires strong energy such as ultraviolet light. Moreover, indirect methods via activation of expensive transition metal-based photocatalysts with visible light response are frequently used. ${ }^{2}$ Photochemical reactions using electron donor-acceptor (EDA) complexes, a new type of non-covalent bonding proposed by Mulliken, have recently attracted much attention as an alternative methodology to photocatalysis. ${ }^{3}$ EDA complexes are formed by the coordination between two molecules, such as electron donors and electron acceptors. In many cases, the energy of this transition is in the visible light region, and one electron is transferred from the electron donor to the electron acceptor, leading to a reactive open-shell intermediate (Figure 1a). ${ }^{4}$ Various aggregation modes of EDA complexes are reported, and $\pi-\pi$ interactions and hydrogen bonds are frequently used in the organic photochemistry, but recently $n-\sigma$ interactions such as halogen-bonding interactions have been attracting attention. ${ }^{5}$ 
Halogen-bonding interactions compared with other intermolecular non-covalent interactions such as hydrogen bonding possess an advantage: the halogen-bond interaction has a greater directionality mainly, due to which, the positive electrostatic potential region, $\sigma$-hole, is located at the center of the $\mathrm{C}$-X axis, resulting in a nearly linear structure with an angle of $160^{\circ}$ to $180^{\circ} .^{6}$ Moreover, since the photochemical activation of EDA complex through halogen-bonding interaction is the aggregation between the organic halide and the electron donor, selective activation of the organic halide and generation of radical carbon species only by fragmentation of the anion radical species generated are potentially possible. (Figure $1 \mathrm{~b}) .^{5}$

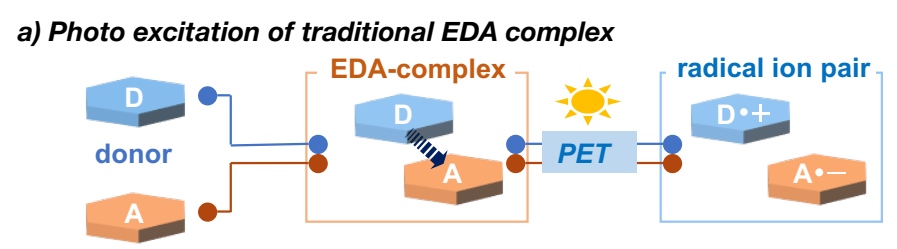

b) Photo excitation of EDA complex via halogen-bonding interaction

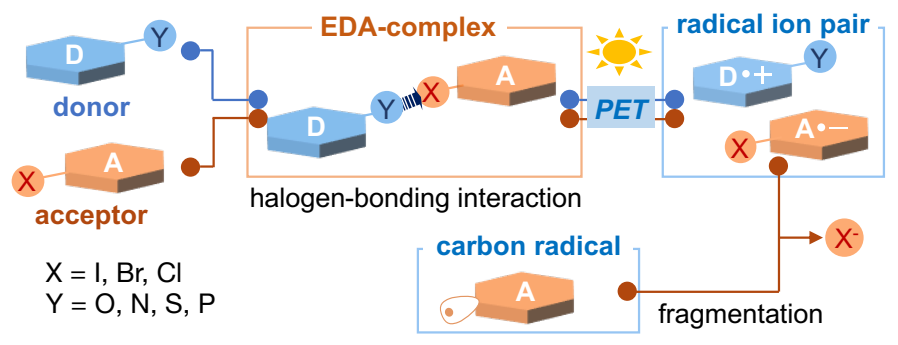

Figure 1. (a) Classical EDA complex theory. (b) A halogen-bonding strategy to produce $C$-centered radical species. PET: photo-induced electron transfer.

Arylboronic esters have been recognized as useful reagents and building blocks in contemporary synthetic organic chemistry due to many catalytic $\mathrm{C}-\mathrm{C}$ bond formations, such as Suzuki-Miyaura cross-coupling. ${ }^{7}$ The classical method for the preparation of aryl boronates is the reaction using pyropholic lithium reagents (RLi) or Grignard reagents $(\mathrm{RMgX})$ with boron-based electrophiles (Scheme 1a). ${ }^{8}$ On the contrary, the transition-metal-catalyzed borylation reaction as an alternative approach to constructing $\mathrm{C}(\mathrm{sp} 2)-\mathrm{B}$ bond has been developed from haloarenes and aromatic hydrocarbons as the corresponding boronic acids/esters. ${ }^{9-15}$ These are robust methodologies to produce organoboron compounds. However, they require expensive transition metal catalysts and high temperatures. Hence, there has been much interest in developing alternative methods using photoreaction in recent years. ${ }^{16}$ For example, the "UV light" induced borylation of haloarenes using quaternary aryl ammonium salts under transition metal-free conditions was reported by $\mathrm{Li}$ et al. ${ }^{17}$ and Larionov et al. ${ }^{18}$ Moreover, Jiao and coworkers reported a noble organocatalytic reaction for the borylation of unactivated aryl chlorides by photoactivation of $i n$ situ-generated SED (single electron donor) complex. ${ }^{19}$ The key to this photo-induced borylation is the formation of aryl radicals. 
Therefore, we estimated that developing a simple and rapid methodology for generating aryl radicals would lead to novel photo-induced borylation.

We have recently developed the use of an in situ-generated halogen-bonding complex to enable the photo-induced transformation of olefins via ATRA reactions. ${ }^{20}$ Therefore, inspired by the methods, we started to challenge the development of the direct borylation of aryl halides using halogen-bonding interaction (Scheme 1b). Herein, we report a photochemical $\mathrm{C}-\mathrm{X}$ bond borylation reaction using novel aryl radical generation strategy through the halogen-bonding EDA complex.

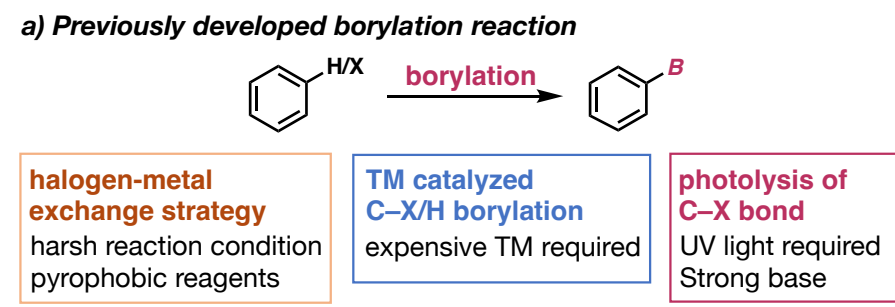

b) This work: $C-X$ borylation by halogen-bonding activation mode

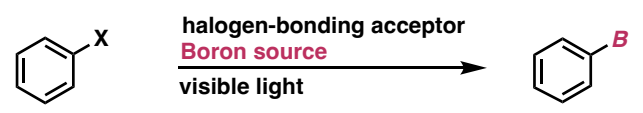

Scheme 1. (a) Traditional borylation tachnology. (b) This work: photo-induced C-X borylation mediated by halogen-bonding complex.

\section{RESULTS AND DISCUSSION}

To validate our proposal, we studied the radical borylation reaction of haloarenes 1. Present experiments were conducted in acetonitrile (MeCN) using commercially available bis (pinacolato) diboron 2a as a cheap and stable boron source. Our overarching goal was to define a strategy that permits the predictable and mild activation of substrates that would be inert to other practical approaches, including photoredox catalysis. Therefore, 4iodoanisole 1a, which is difficult to activate by photocatalysis reductively, was selected as the precursor of aryl radicals. ${ }^{21}$ After carefully screening the conditions, it was found that the optimal reaction conditions include the use of a $3 \mathrm{~W} 420 \mathrm{~nm}$ LED as the light source, $\mathrm{MeCN}$ as the solvent, and performing the reaction at room temperature under Ar atmosphere to furnish the desired borylation product $\mathbf{4 a}$ in $92 \%$ yield (Table 1, entry 1 ). ${ }^{22}$ According to the base screening results, we found that $\mathrm{NaOH}$ and $\mathrm{KOH}$ were also an efficient base for the photochemical $\mathrm{C}-\mathrm{I}$ borylation reaction (entry 1 vs. entries 2-5). Other solvents, such as THF, $\mathrm{MeOH}$, and DMF, were proved inferior (entries 6-8). Control experiments revealed the essential role of $\mathrm{K}_{2} \mathrm{CO}_{3}, 2-\mathrm{NpOH}$, and photoirradiation on the observed reactivity (entries 9-11). The desired borylated product $4 \mathrm{a}$ was formed when the reaction was under an oxygen atmosphere, albeit in a slightly low yield (entry 12). Finally, when a catalytic amount of 2-NpOH was employed, the borylation reaction proceeded catalytically, albeit the yield of the desired product decreased (entry 13). 
Table 1. Optimization study for $\mathrm{C}-\mathrm{I}$ borylation reaction of $\mathbf{1} \mathbf{a}^{\mathrm{a}}$.

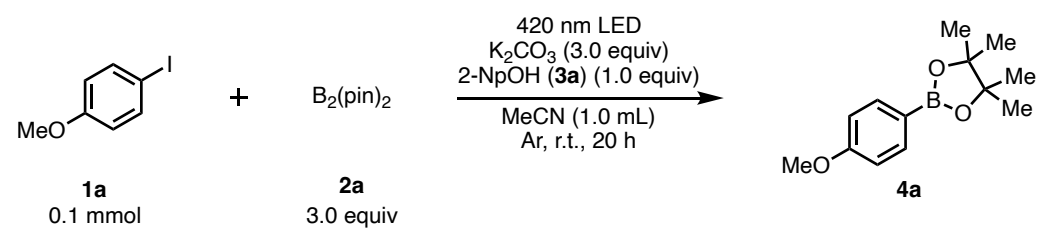

$\begin{array}{ccc}\text { Entry } & \text { Changed from standard conditions } & \text { Yield (\%) } \\ 1 & - & 95(92) \\ 2 & \mathrm{Na}_{2} \mathrm{CO}_{3} \text { instead of } \mathrm{K}_{2} \mathrm{CO}_{3} & 10 \\ 3 & \mathrm{Cs}_{2} \mathrm{CO}_{3} \text { instead of } \mathrm{K}_{2} \mathrm{CO}_{3} & 87 \\ 4 & \mathrm{NaOH} \text { instead of } \mathrm{K}_{2} \mathrm{CO}_{3} & 94 \\ 5 & \mathrm{KOH} \text { instead of } \mathrm{K}_{2} \mathrm{CO}_{3} & 93 \\ 6 & \mathrm{THF} \text { instead of } \mathrm{MeCN} & 10 \\ 7 & \mathrm{MeOH} \text { instead of } \mathrm{MeCN} & 29 \\ 8 & \mathrm{DMF} \text { instead of } \mathrm{MeCN} & 50 \\ 9 & \text { w/o } \mathrm{K}_{2} \mathrm{CO}_{3} & 3 \\ 10 & \text { w/o 2-NpOH }(3 \mathbf{3 a}) & 10 \\ 11 & \text { dark or dark at } 50{ }^{\circ} \mathrm{C} & \text { trace } \\ 12 & \text { under } \mathrm{O}_{2} & 64 \\ 13 & \text { 3a }(0.1 \text { equiv) } & 64\end{array}$

${ }^{\mathrm{a}}$ Yield was determined by ${ }^{1} \mathrm{H}$ NMR analysis of crude reaction mixture using 1,1,2,2 tetrachloroethane as an internal standard. The number in parenthesis is isolated yield.

Further, we explored the scope of the photo-induced borylation using optimized reaction conditions (Figure 2). Iodoarenes with electron-donating substituents, regardless of the substitution pattern, were converted to the corresponding arylboronic esters in moderate to excellent yields (4a-g). Moreover, a scale-up experiment was also conducted to furnish corresponding boronate (4a) ester in $81 \%$ yield. The sterically hindered substrates $\mathbf{1 h}$ and $\mathbf{1 i}$ reacted well to give arylboronate $\mathbf{4 h}$ and $\mathbf{4 i}$ in moderate yields. Naturally, the unsubstituted haloarene $\mathbf{1 j}$ reacted sufficiently to obtain the desired product $\mathbf{4 j}$. Additionally, we have shown that bromoarene $\mathbf{2} \mathbf{j}$ can also be adapted using a strong base, albeit in moderate boronate ester yield $\left(\mathrm{KO}^{t} \mathrm{Bu}\right)$. Borylation of halogenated iodoarenes bearing fluoro (4k), chloro (4I), bromo (4m), and iodo (4n) groups was also demonstrated. Fluorine substituents with weak ability as halogen-bonding donors gave the corresponding borylated products in good yields, but for chlorine-, bromine-, and iodine-substituted substrates, undesired diborylation reactions proceeded, and the yields significantly decreased. Unfortunately, although haloarenes with strong electron-deficient substituents, such as $\mathrm{CF}_{3}$ groups $(\mathbf{4 u}$ and $\mathbf{4 v}$ ) are known to be good halogen-bonding donors, the borylation reaction did not proceed properly under 
optimal reaction conditions. Furthermore, aryl halides with carbonyls (4q-t), such as ketones, aldehydes, esters, and cyanides, which are unstable under basic conditions, gave the desired borylated products (5q-t) without decomposition in any case under the reaction conditions. ${ }^{19}$ It should be noted that the borylation reaction proceeded smoothly even with relatively inert bromoarenes. Interestingly, in the case of 4-halo benzaldehyde (4s), the reaction proceeds without adding phenol as a halogen-bonding acceptor, suggesting that the $\mathrm{C}-\mathrm{X}$ bond's direct hemolysis may proceed a different pathway for this substrate. ${ }^{23}$ Substrates with protected or unprotected hydroxyl or amino groups could be converted smoothly to the corresponding borylation products (4u-4ai). Although these substrates (1u-1ai) may interfere with the halogen-bond formation, they were used in this reaction without yield loss. The fact that unprotected phenols and anilines can be directly converted to boronates suggests that the reaction is highly valuable. Excellent yields were obtained for the biphenyl and naphthyl substrate (4aj-4al). The halogen-bonding interaction initiated the borylation reaction of aromatic halide, promoted by phenol $\mathbf{2}$, provided a range of borylated heteroarenes in moderate to excellent yield (4am-4au). Additionally, the borylated substrate (B(pin); 1av and $\mathrm{B}(\mathrm{dan})$; 1aw) also applied to the present borylation; the C-I bond was converted to C-B bond in $92 \%$ for $4 \mathbf{a v}$ and $60 \%$ for $4 \mathrm{aw}$, respectively.

Further experiments demonstrated that this method is compatible with the functionalization of biorelevant compounds. It allowed the late-stage introduction of boronate ester into menthol-derived aryl iodide (1ax) and protected glucose-derived aryl iodide (1ay) in a single step. Finally, we applied our strategy to perform other boron sources, such as bis (neopentyl glycolato) diboron (2b) and bis (hexylene glycolato) diboron (2c). This transformation, using other boron sources, afforded the corresponding borylated product (5a and $\mathbf{5 b})$ in excellent yield. However, the use of tetrahydroxydiboron resulted in no reaction, which is suspected to be due to the acidbase reaction between diboron and the base proceeded. 


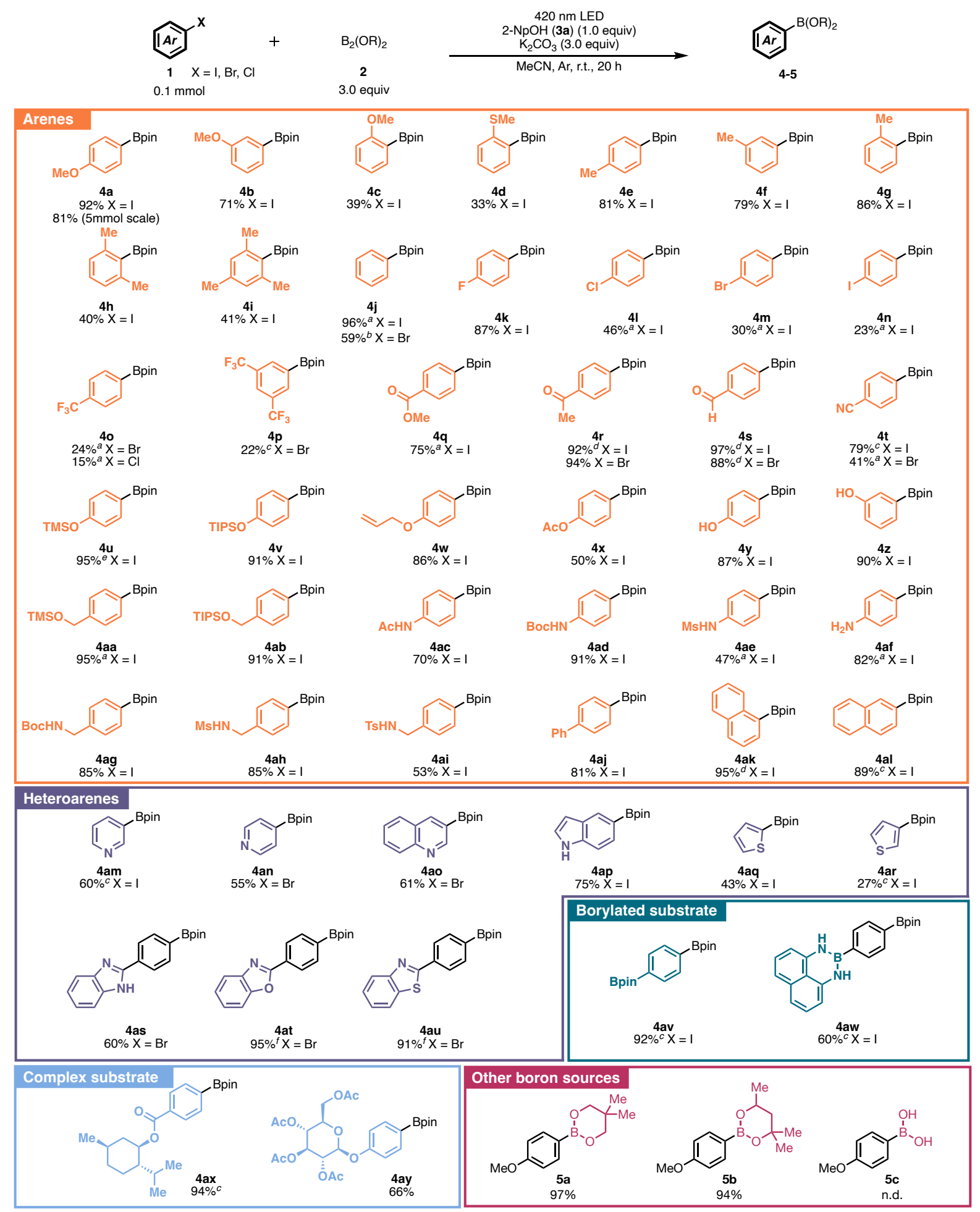

Figure 2. Substrate scope for $\mathbf{C}-\mathbf{I}$ borylation reaction. Reaction conditions: 1, 2, or 3 ( $0.1 \mathrm{mmol}, 1.0$ equiv), diboron reagents (3.0 equiv), $\mathrm{K}_{2} \mathrm{CO}_{3}(3.0$ equiv), $2-\mathrm{NpOH}$ (1.0 equiv) in $1.0 \mathrm{~mL}$ of MeCN, irradiated under $420 \mathrm{~nm}$ LED at room temperature for $20 \mathrm{~h}$. Yields of isolated products are reported. ${ }^{a} 1-\mathrm{NpOH}$ (1.0 equiv) was used instead of 2-NpOH. ${ }^{b} \mathrm{KO}{ }^{t} \mathrm{Bu}$ (1.0 equiv) was used instead of $\mathrm{K}_{2} \mathrm{CO}_{3} \cdot{ }^{c} 2,6-\mathrm{Cl}_{2}-\mathrm{C}_{6} \mathrm{H}_{3} \mathrm{OH}$ (1.0 equiv) was used instead of 2- 
NpOH. ${ }^{d}$ Without 2-NpOH. ${ }^{e}$ After column chromatography, the deprotected product was isolated. ${ }^{24 f} 4-\mathrm{Ph}-\mathrm{C}_{6} \mathrm{H}_{4} \mathrm{OH}$ (1.0 equiv) was used instead of $2-\mathrm{NpOH}$.

Several controlled experiments were carried out (Scheme 3). We hypothesize that the aryl radicals are generated by forming EDA complexes and irreversible fragmentation through PET, as shown in Scheme 3a. First, the effect of phenolic hydroxyl groups was investigated (Scheme 3b). The reaction using preformed potassium naphthoxide gave the corresponding boronate (4a). In contrast, the reaction did not proceed when corresponding methylprotected naphthol was used. This is indicated that the phenoxide formed from phenol with base in the reaction mixture may have promoted the reaction. We also discovered that 2-NpOK was used instead of 2-NpOH in $\mathrm{MeCN}$, showing a significant redshift to the visible region when preformed. The shoulder peak was observed at approximately 420-450 nm (Scheme 3b, blue line vs. gray line). The addition of iodobenzene did not affect their UV absorption (Scheme 3b, orange line). Moreover, we observed the formation of a 1:1 complex between 2naphtoxide and 1a with a binding constant $(K a)$ of $0.99 \mathrm{M}^{-1}$ in DMSO- $d_{6}$ using a ${ }^{1} \mathrm{H}$ NMR titration method and Job's plot analysis (Scheme 3c, See ESI, Figure S1-4). Since these results are similar to the previous results ${ }^{5 \mathrm{e}}$ and are sufficient to rule out the formation of EDA complexes by $\pi-\pi *$ interaction or $n-\pi *$ interaction, such as the independent reporting by Miyake and Melchior, ${ }^{25,26}$ we hypothesize that the formation of EDA complexes by halogen-bonding interactions is the key to initiating the reaction.

We performed several controlled experiments to determine aryl radical generation through PET after forming EDA complexes through halogen-bonding interaction (Scheme 3d). When the reaction using 2-iodo-allylphenol $1 \mathbf{a z}$ was used as a substrate, borylation reaction proceeded after radical cyclization reaction to furnish $\mathbf{4 a z}$ in $39 \%$ yield (Scheme 3d, eq. 1). Moreover, methyl 2-iodobenzoate 1 ba to the reaction resulted in no desired product formation and gave the $\mathrm{C}-\mathrm{C}$ and $\mathrm{C}-\mathrm{O}$ forming cyclic product 6 in $11 \%$ yield (Scheme 3d, eq. 2). This byproduct is thought to be formed by a similar pathway as that using hypervalent iodine compounds by Miyake. ${ }^{25}$ Therefore, the aryl radical intermediate formed after PET reacts with the phenoxy radical to form a $\mathrm{C}-\mathrm{C}$ bond. The byproduct can be obtained by rearrangement and removal of water. ${ }^{27}$ When the reaction was subjected to the optimized condition with the addition of radical scavengers, such as TEMPO, the desired product formation was significantly suppressed; instead, the formation of TEMPO adducts of aryl radicals $7 \mathbf{a}$ was obtained (Scheme 3d, eq. 3). ${ }^{28}$ At the same time, $7 \mathrm{~b}$ is formed by the reaction of TEMPO with the acetonitrile radical generated by the hydrogen atom abstraction from acetonitrile by the phenoxy radical generated in situ. ${ }^{29}$ 


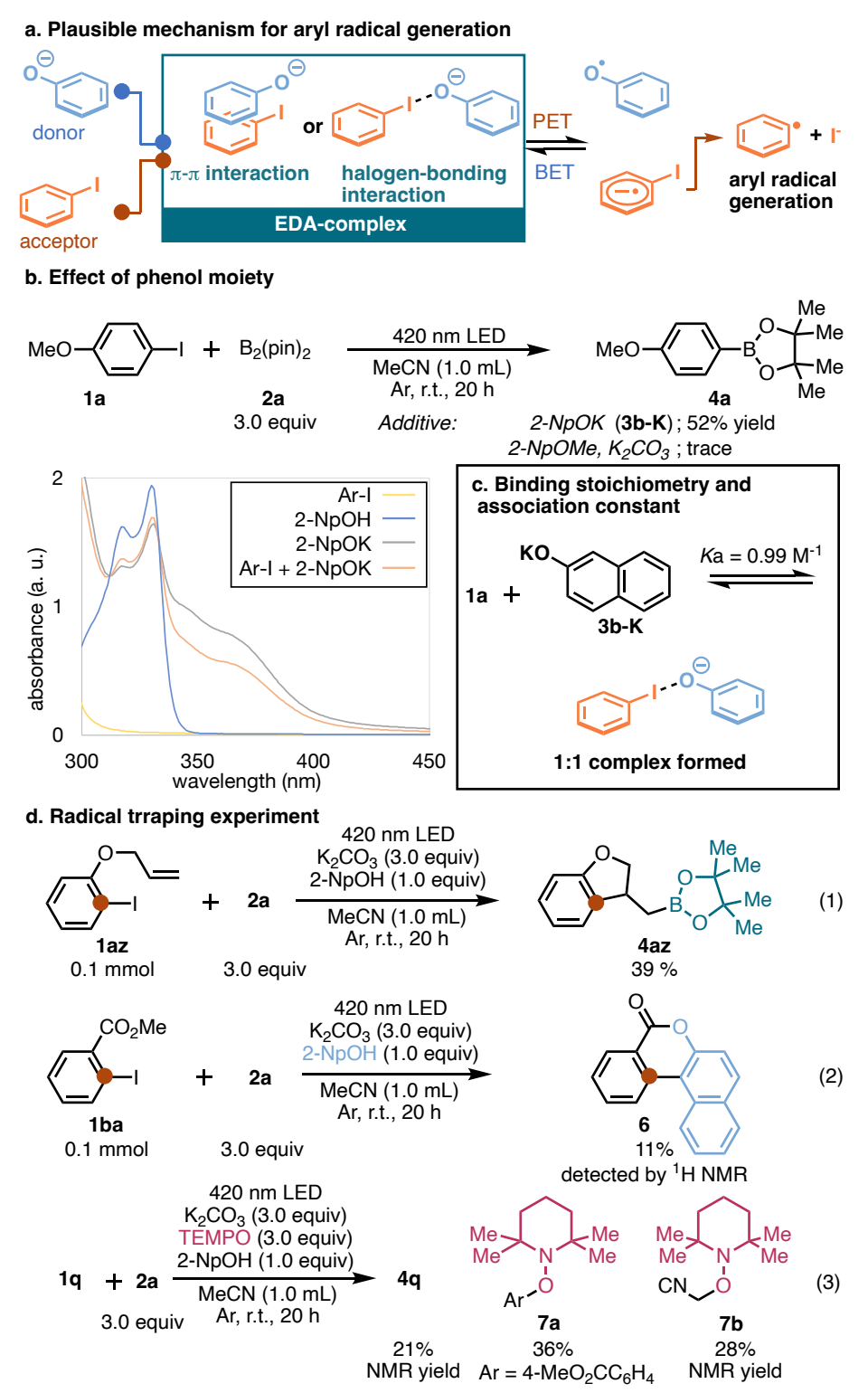

Scheme 3. Controlled experiments. (a) A plausible mechanism for aryl radical generation step. (b) The reaction employs potassium 2-naphtoxide and 2-MeO-Np and UV/Vis spectrum. (c) Investigation of binding stoichiometry and association constant. (d) Intermolecular and intramolecular radical trapping experiments. ${ }^{a}$ Isolated yield.

Based on the previously reported results and our experimental evidence, the possible reaction pathway for geeeranting the aryl radical is shown in Scheme $4 .^{5 e, 25,30,31 .}$ The reaction was initiated by photoexcitation of the halogen-bonding complex A to produce the excited state $\mathbf{B}$ under irradiation of $420 \mathrm{~nm}$ LED. The photoexcitation of B undergoes ET to an aryl halide, producing an aryl radical anion $\mathbf{C}$ and phenoxy radical. After the fragmentation of $\mathbf{C}$, the generated aryl radical $\mathbf{D}$ reacts with the in situ formed borate $\mathbf{E}$ to produce an arylboronate $\mathbf{4}$ and boryl radical anion $\mathbf{F}{ }^{31}$ The radical anion $\mathbf{F}$ can also be viewed as an anionic based stabilized boryl radical. Finally, $\mathbf{F}$ is oxidized by the phenoxy radical to form the borate $\mathbf{G}$. 


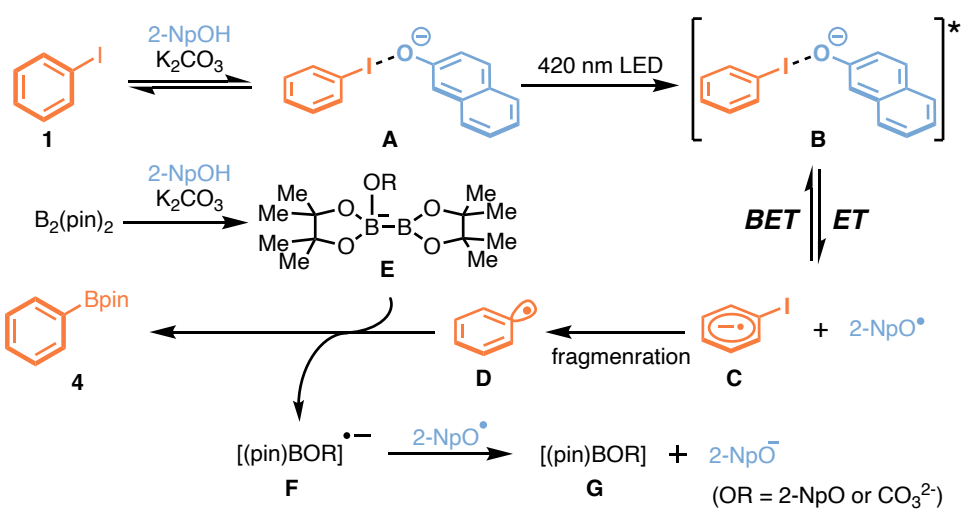

Scheme 4. Plausible reaction mechanism for photo-induced borylation reaction aryl halide.

\section{CONCLUSION}

In summary, the photo-induced borylation of aryl halides promoted by phenol derivatives via halogen bonding was investigated. The borylation of aryl halides with $\mathrm{B}_{2}(\mathrm{pin})_{2}$ afforded various products in moderate to good yields when irradiated at $420 \mathrm{~nm}$. The photoactivated borylation from our study revealed that an EDA complex between aryl halides and phenoxides was a crucial intermediate in the subsequent charge separation processes that led to the formation of aryl radical. Furthermore, this is the first reported example of a methodology using phenol as an acceptor for the halogen-bond. Further efforts are focused on extending this new methodology to other classes of compounds for photocatalysis, including inactive aryl halides or alkyl halides.

\section{-ASSOCIATED CONTENT}

\section{Supporting Information}

The Supporting Information is available free of charge at@@@@.1

$\mathrm{H},{ }^{13} \mathrm{C}\left\{{ }^{1} \mathrm{H}\right\}$ and ${ }^{19} \mathrm{~F}$ NMR spectra, UV/Vis spectra, results of full detailed optimization study experiments, and characterization of all compounds synthesized.

\section{-AUTHOR INFORMATION}

\section{Corresponding Author}

Correspondence to E.Y. and A.I. 
Eiji Yamaguchi (yamaguchi@gifu-pu.ac.jp)

Akichika Itoh (itoha@gifu-pu.ac.jp)

\section{Competing interests}

The authors declare no competing interests.

\section{Acknowledgments}

This work was supported by the Takeda Science Foundation, and JSPS KAKENHI Grant number: 20K06968.

\section{References}

(1) (a) Roth, H. D. The Beginnings of Organic Photochemistry. Angew. Chem. Int. Ed. 1989, 28, 11931207. (b) Boyd, M. K.; Zopp, G. M. Organic Photochemistry. Annu. rep. prog. chem., Sect. B. 2004, 100, 351-375. (c) Hoffmann, N. Photochemical Reactions as Key Steps in Organic Synthesis.Chemical Reviews. 2008, 108, 1052-1103. (d) Knowles, J. P.; Elliott, L. D.; BookerMilburn, K. I. Flow Photochemistry: Old Light through New Windows. Beilstein J. Org. Chem. 2012, 8, 2025-2052. (e) Beeler, A. B. Introduction: Photochemistry in Organic Synthesis. Chem. Rev. 2016, 116, 9629-9630. (f) Sambiagio, C.; Noël, T. Flow Photochemistry: Shine Some Light on Those Tubes! Trends Chem. 2020, 2, 92-106. (g) Bonfield, H. E.; Knauber, T.; Lévesque, F.; Moschetta, E. G.; Susanne, F.; Edwards, L. J. Photons as a 21st Century Reagent. Nature Commun. 2020, $11,804$.

(2) (a) Prier, C. K.; Rankic, D. A.; MacMillan, D. W. C. Visible Light Photoredox Catalysis with Transition Metal Complexes: Applications in Organic Synthesis. Chem. Rev. 2013, 113, 5322-5363. (b) Iers, F. T. I Norgan I C Chemistry. Inorg. Chem. Front. 2015, 2, 687-712. (c) Shaw, M. H.; Twilton, J.; MacMillan, D. W. C. Photoredox Catalysis in Organic Chemistry. J. Org. Chem. 2016, 81, 6898-6926. (d) Liu, J.; Lu, L.; Wood, D.; Lin, S. New Redox Strategies in Organic Synthesis by Means of Electrochemistry and Photochemistry. ACS Cent. Sci. 2020, 6, 1317-1340. (e) Crisenza, G. E. M.; Melchiorre, P. Chemistry Glows Green with Photoredox Catalysis. Nat. Commun. 2020, 11, 803. (f) Melchionna, M.; Fornasiero, P. Updates on the Roadmap for Photocatalysis. ACS Catal. 2020, 10, 5493-5501.

(3) (a) Mulliken, R. S. Molecular Compounds and Their Spectra. II. J. Am. Chem. Soc. 1952, 74, 811824. (b) Mulliken, R. S. Molecular Compounds and Their Spectra. III. The Interaction of Electron Donors and Acceptors. J. Phys. Chem. 1952, 56, 801-822. 
(4) (a) Lima, C. G. S.; Lima, T. D. M.; Duarte, M.; Jurberg, I. D.; Paixão, M. W. Organic Synthesis Enabled by Light-Irradiation of EDA Complexes: Theoretical Background and Synthetic Applications. ACS Catal. 2016, 6, 1389-1407. (b) Crisenza, G. E. M.; Mazzarella, D.; Melchiorre, P. Synthetic Methods Driven by the Photoactivity of Electron Donor-Acceptor Complexes. J. Am. Chem. Soc. 2020, 142, 5461-5476. (c) Yang, Z.; Liu, Y.; Cao, K.; Zhang, X.; Jiang, H.; Li, J. Synthetic Reactions Driven by Electron-Donor-Acceptor(EDA) Complexes. Beilstein J. Org. Chem. 2021, 17, 771-799.

(5) (a) Wang, Y.; Wang, J.; Li, G. X.; He, G.; Chen, G. Halogen-Bond-Promoted Photoactivation of Perfluoroalkyl Iodides: A Photochemical Protocol for Perfluoroalkylation Reactions. Org. Lett. 2017, 19, 1442-1445. (b) Postigo, A. Electron Donor-Acceptor Complexes in Perfluoroalkylation Reactions. European J. Org. Chem. 2018, 2018, 6391-6404. (c) Miao, R.; Wang, D.; Xiao, J.; Ma, J.; Xue, D.; Liu, F.; Fang, Y. Halogen Bonding Matters: Visible Light-Induced Photoredox CatalystFree Aryl Radical Formation and Its Applications. Phys. Chem. Chem. Phys. 2020, 22, 10212-10218. (d) Nandy, A.; Kazi, I.; Guha, S.; Sekar, G. Visible-Light-Driven Halogen-Bond-Assisted Direct Synthesis of Heteroaryl Thioethers Using Transition-Metal-Free One-Pot C-I Bond Formation/C-S Cross-Coupling Reaction. J. Org. Chem. 2021, 86, 2570-2581. (d) Sundaravelu, N.; Nandy, A.; Sekar, G. Visible Light Mediated Photocatalyst Free C-S Cross Coupling: Domino Synthesis of Thiochromane Derivatives via Photoinduced Electron Transfer. Org. Lett. 2021, 23, 3115-3119. (e) Li, T.; Liang, K.; Tang, J.; Ding, Y.; Tong, X.; Xia, C. A Photoexcited Halogen-Bonded EDA Complex of the Thiophenolate Anion with Iodobenzene for $\mathrm{C}\left(\mathrm{sp}^{3}\right)-\mathrm{H}$ Activation and Thiolation. Chem. Sci. 2021, 12, 15655-15661.

(6) (a) Sarwar, M. G.; Dragisic, B.; Salsberg, L. J.; Gouliaras, C.; Taylor, M. S. Thermodynamics of Halogen Bonding in Solution: Substituent, Structural, and Solvent Effects. J. Am. Chem. Soc. 2010, 132, 1646-1653. (b) Zhou, F.; Han, J.; Liu, R.; Li, P.; Zhang, H. Charge-Transfer Complexes of Iodoform with 1,4-Dioxane, -Dithiane, and -Diselenane: Theoretical Electron Density and Energy Decomposition Analysis. Comput. Theor. Chem. 2014, 1044, 80-86. (c) Cavallo, G.; Metrangolo, P.; Milani, R.; Pilati, T.; Priimagi, A.; Resnati, G.; Terraneo, G. The Halogen Bond. Chem. Rev. 2016, 116, 2478-2601. (d) Maugeri, L.; Jamieson, E. M. G.; Cordes, D. B.; Slawin, A. M. Z.; Philp, D. PH Controlled Assembly of a Self-Complementary Halogen-Bonded Dimer. Chem. Sci. 2017, 8, 938-945. (e) Bracker, M.; Helmecke, L.; Kleinschmidt, M.; Czekelius, C.; Marian, C. M. Visible Light-Induced Homolytic Cleavage of Perfluoroalkyl Iodides Mediated by Phosphines. Molecules 2020, 25, 1-18.

(7) Palladium-Catalyzed Cross-Coupling Reactions of Organoboron Compounds. Chem. Rev. 1995, 95, $2457-2483$. 
(8) (a) Brown, H. C.; Srebnik, M.; Cole, T. E. Organoboranes. 48. Improved Procedures for the Preparation of Boronic and Borinic Esters. Organometallics 1986, 5, 2300-2303. (b) Matteson, D. S. Boronic Esters in Stereodirected Synthesis. Tetrahedron 1989, 45, 1859-1885.

(9) Selected examples for Fe-catalyzed borylation; (a) Yoshida, T.; Ilies, L.; Nakamura, E. IronCatalyzed Borylation of Aryl Chlorides in the Presence of Potassium t-Butoxide. ACS Catal. 2017, 7, 3199-3203. (b) Bedford, R. B.; Brenner, P. B.; Carter, E.; Gallagher, T.; Murphy, D. M.; Pye, D. R. Iron-Catalyzed Borylation of Alkyl, Allyl, and Aryl Halides: Isolation of an Iron(I) Boryl Complex. Organometallics 2014, 33, 5940-5943.

(10) Selected examples for Co-catalyzed borylation; (a) Yao, W.; Fang, H.; Peng, S.; Wen, H.; Zhang, L.; Hu, A.; Huang, Z. Cobalt-Catalyzed Borylation of Aryl Halides and Pseudohalides. Organometallics 2016, 35, 1559-1564. (b) Komeyama, K.; Kiguchi, S.; Takaki, K. The Drastic Effect of Cobalt and Chromium Catalysts in the Borylation of Arylzinc Reagents. Chem. Commun. 2016, 52, 7009-7012. (c)Verma, P. K.; Mandal, S.; Geetharani, K. Efficient Synthesis of Aryl Boronates via Cobalt-Catalyzed Borylation of Aryl Chlorides and Bromides. ACS Catal. 2018, 8 , 4049-4054.

(11) Selected examples for Ni-catalyzed borylation; Liu, X. W.; (a) Rosen, B. M.; Huang, C.; Percec, V. Sequential Ni-Catalyzed Borylation and Cross-Coupling of Aryl Halides via in Situ Prepared Neopentylglycolborane. Org. Lett. 2008, 10, 2597-2600. (b) Moldoveanu, C.; Wilson, D. A.; Wilson, C. J.; Leowanawat, P.; Resmerita, A. M.; Liu, C.; Rosen, B. M.; Percec, V. Neopentylglycolborylation of Ortho -Substituted Aryl Halides Catalyzed by $\mathrm{NiCl}_{2}$-Based MixedLigand Systems. J. Org. Chem. 2010, 75, 5438-5452. (c) Yamamoto, T.; Morita, T.; Takagi, J.; Yamakawa, T. $\mathrm{NiCl}_{2}\left(\mathrm{PMe}_{3}\right)_{2}$-Catalyzed Borylation of Aryl Chlorides. Org. Lett. 2011, 13, 57665769. (d) Echavarren, J.; Zarate, C.; Martin, R. Ni-Catalyzed Borylation of Aryl Fluorides via C-F Cleavage. J. Am. Chem. Soc. 2015, 137, 12470-12473.

(12) Selected examples for Cu-catalyzed borylation; (a) Zhu, W.; Ma, D. Formation of Arylboronates by a Cul-Catalyzed Coupling Reaction of Pinacolborane with Aryl Iodides at Room Temperature. Org. Lett. 2006, 8, 261-263. (b) Kleeberg, C.; Dang, L.; Lin, Z.; Marder, T. B. A Facile Route to Aryl Boronates: Room-Temperature, Copper-Catalyzed Borylation of Aryl Halides with Alkoxy Diboron Reagents. Angew. Chem. Int. Ed. 2009, 48, 5350-5354. (c) Labre, F.; Gimbert, Y.; Bannwarth, P.; Olivero, S.; Duñach, E.; Chavant, P. Y. Application of Cooperative Iron/Copper Catalysis to a Palladium-Free Borylation of Aryl Bromides with Pinacolborane. Org. Lett. 2014, 16, 2366-2369. (d) Kuehn, L.; Huang, M.; Radius, U.; Marder, T. B. Copper-Catalysed Borylation of Aryl Chlorides. Org. Biomol. Chem. 2019, 17, 6601-6606.

(13) Selected examples for Zn-catalyzed borylation; Nagashima, Y.; Takita, R.; Yoshida, K.; Hirano, K.; Uchiyama, M. Design, Generation, and Synthetic Application of Borylzincate: Borylation of Aryl Halides and Borylzincation of Benzynes/Terminal Alkyne. J. Am. Chem. Soc. 2013, 135, 18730- 
18733. (b) Bose, S. K.; Marder, T. B. Efficient Synthesis of Aryl Boronates via Zinc-Catalyzed Cross-Coupling of Alkoxy Diboron Reagents with Aryl Halides at Room Temperature. Org. Lett. 2014, 16, 4562-4565. (c) Bose, S. K.; Deißenberger, A.; Eichhorn, A.; Steel, P. G.; Lin, Z.; Marder, T. B. Zinc-Catalyzed Dual C-X and C-H Borylation of Aryl Halides. Angew. Chem. Int. Ed. 2015, 54, 11843-11847. (d) Li, Y.; Dang, Y.; Li, D.; Pan, H.; Zhang, L.; Wang, L.; Cao, Z.; Li, Y. Zinc Complexes with an Ethylene-Bridged Bis( $\beta$-Diketiminate) Ligand: Syntheses, Structures, and Applications as Catalysts in the Borylation of Aryl Iodides. Organometallics 2021, 40, 482-489.

(14) Selected examples for Pd-catalyzed borylation; (a) Ishiyama, T.; Murata, M.; Miyaura, N. Palladium(0)-Catalyzed Cross-Coupling Reaction of Alkoxydiboron with Haloarenes: A Direct Procedure for Arylboronic Esters. J. Org. Chem. 1995, 60, 7508-7510. (b) Billingsley, K. L.; Barder, T. E.; Buchwald, S. L. Palladium-Catalyzed Borylation of Aryl Chlorides: Scope, Applications, and Computational Studies. Angew. Chem. Int. Ed. 2007, 46, 5359-5363. (c) Billingsley, K. L.; Buchwald, S. L. An Improved System for the Palladium-Catalyzed Borylation of Aryl Halides with Pinacol Borane. J. Org. Chem. 2008, 73, 5589-5591. (d) Kawamorita, S.; Ohmiya, H.; Iwai, T.; Sawamura, M. Palladium-Catalyzed Borylation of Sterically Demanding Aryl Halides with a SilicaSupported Compact Phosphane Ligand. Angew. Chem. Int. Ed. 2011, 50, 8363-8366. (e) Bej, A.; Srimani, D.; Sarkar, A. Palladium Nanoparticle Catalysis: Borylation of Aryl and Benzyl Halides and One-Pot Biaryl Synthesis via Sequential Borylation-Suzuki-Miyaura Coupling. Green Chem. 2012, 14, 661-667.

(15) Selevted review for Ir-catalyzed borylation; Hartwig, J. F. Borylation and Silylation of C-H Bonds: A Platform for Diverse C-H Bond Functionalizations. Acc. Chem. Res. 2012, 45, 864-873.

(16) Selevted review for photo-induced borylation; Yadagiri, B.; Daipule, K.; Singh, S. P. Photoinduced Borylation Reactions: An Overview. Asian J. Org. Chem. 2021, 10, 7-37.

(17) Chen, K.; Zhang, S.; He, P.; Li, P. Efficient Metal-Free Photochemical Borylation of Aryl Halides under Batch and Continuous-Flow Conditions. Chem. Sci. 2016, 7, 3676-3680.

(18) Mfuh, A. M.; Doyle, J. D.; Chhetri, B.; Arman, H. D.; Larionov, O. V. Scalable, Metal- and Additive-Free, Photoinduced Borylation of Haloarenes and Quaternary Arylammonium Salts. J. Am. Chem. Soc. 2016, 138, 2985-2988.

(19) Zhang, L.; Jiao, L. Visible-Light-Induced Organocatalytic Borylation of Aryl Chlorides. J. Am. Chem. Soc. 2019, 141, 9124-9128.

(20) (a) Matsuo, K.; Yamaguchi, E.; Itoh, A. In Situ-Generated Halogen-Bonding Complex Enables Atom Transfer Radical Addition (ATRA) Reactions of Olefins. J. Org. Chem. 2020, 85, 1057410583. (b) Matsuo, K.; Kondo, T.; Yamaguchi, E.; Itoh, A. Photoinduced Atom Transfer Radical Addition Reaction of Olefins with $\alpha$-Bromo Carbonyls. Chem. Pharm. Bull. 2021, 69, 796-801. (c) Matsuo, K.; Yoshitake, T.; Yamaguchi, E.; Itoh, A. Photoinduced Atom Transfer Radical 
Addition/Cyclization Reaction between Alkynes or Alkenes with Unsaturated $\alpha$-Halogenated Carbonyls. Molecules 2021, 26, 6781.

(21) Zhang, L.; Jiao, L. Super Electron Donors Derived from Diboron. Chem. Sci. 2018, 9, 2711-2722.

(22) See ESI for full detailed optimization study as Table S1-3.

(23) Cheng, Y.; Mück-Lichtenfeld, C.; Studer, A. Metal-Free Radical Borylation of Alkyl and Aryl Iodides. Angew. Chem. Int. Ed. 2018, 57, 16832-16836.

(24) Yamamoto, Y.; Matsubara, H.; Yorimitsu, H.; Osuka, A. Base-Free Palladium-Catalyzed Borylation of Aryl Chlorides with Diborons. ChemCatChem 2016, 8, 2317-2320.

(25) Liu, B.; Lim, C. H.; Miyake, G. M. Light-Driven Intermolecular Charge Transfer Induced Reactivity of Ethynylbenziodoxol(on)e and Phenols. J. Am. Chem. Soc. 2018, 140, 12829-12835.

(26) Arceo, E.; Jurberg, I. D.; Álvarez-Fernández, A.; Melchiorre, P. Photochemical Activity of a Key Donor-Acceptor Complex Can Drive Stereoselective Catalytic $\alpha$-Alkylation of Aldehydes. Nat. Chem. 2013, 5, 750-756.

(27) Satkar, Y.; Wrobel, K.; Trujillo-González, D. E.; Ortiz-Alvarado, R.; Jiménez-Halla, J. O. C.; Solorio-Alvarado, C. R. The Diaryliodonium(III) Salts Reaction With Free-Radicals Enables OnePot Double Arylation of Naphthols. Front. Chem. 2020, 8, 563470. (b) Qian, X.; Han, J.; Wang, L. Copper-Catalyzed Selective Ortho-Arylations of 2-Naphthol and Phenol Derivatives with Diaryliodonium Salts. Tetrahedron Lett. 2016, 57, 607-610.

(28) Yuan, T.; Zheng, M.; Antonietti, M.; Wang, X. Ceramic Boron Carbonitrides for Unlocking Organic Halides with Visible Light. Chem. Sci. 2021, 12, 6323-6332.

(29) Pan, C.; Yang, C.; Li, K.; Zhang, K.; Zhu, Y.; Wu, S.; Zhou, Y.; Fan, B. Photo-Promoted Decarboxylative Alkylation of $\alpha, \beta$-Unsaturated Carboxylic Acids with $\mathrm{ICH}_{2} \mathrm{CN}$ for the Synthesis of $\beta, \gamma$-Unsaturated Nitriles. Org. Lett. 2021, 23, 7188-7193.

(30) Cheng, Y.; Mück-Lichtenfeld, C.; Studer, A. Metal-Free Radical Borylation of Alkyl and Aryl Iodides. Angew. Chem. Int. Ed. 2018, 57, 16832-16836.

(31) Friese, F. W.; Studer, A. New Avenues for C-B Bond Formation via Radical Intermediates. Chem. Sci. 2019, 10, 8503-8518. 